\title{
Prevalence of Overall and Abdominal Obesity among Adolescent High School Girls in Tabriz, Iran
}

\author{
Rafraf $M^{\mathrm{a}}$, Mohamadi $\mathrm{E}^{\mathrm{b}}$, Gargari BPc \\ a Nutritional Research Center, Department of Community Nutrition, Faculty of Health \& Nutrition, Tabriz \\ University of Medical Sciences, Tabriz, Iran. \\ ${ }^{b}$ Faculty of Health \& Nutrition, Tabriz University of Medical Sciences, Tabriz, Iran. \\ 'Department of Nutrition and Diet Therapy, Nutritional Research Center, Tabriz University of Medical Sciences, \\ Tabriz, Iran
}

\begin{abstract}
Introduction: Obesity is a growing public health problem in both developed and developing societies. The aim of this cross-sectional study was to estimate the prevalence of overall obesity determined by body mass index (BMI) and abdominal obesity identified by waist circumference(WC) percentiles, waist to hip ratio(WHR) and waist to height ratio (WHtR), in adolescent girls attending high school in Tabriz, Iran. Methods: A sample of 985 girls was selected by stepwise random sampling from five districts of Tabriz. Weight, height, WC and hip circumferences $(\mathrm{HiC})$ of the subjects were measured and BMI, WHR and WHtR were calculated. Overweight and obesity were defined according to International BMI Cut-Off Points for adolescents. WC $>90$ th percentile, WHR $>0.80$ and WHtR $\geq 0.50$ were categorized as abdominal obesity. Results: The prevalence rates of obesity and overweight were $2.8 \%$ and $16.4 \%$, respectively. Abdominal obesity defined by WC percentiles, WHR, and WHtR were seen in $3.2 \%, 4.0 \%$, and $18.2 \%$ of subjects, respectively. WHtR determined the highest prevalence of abdominal obesity in subjects compared to WC and WHR. WC, WHR, and WHtR were significantly related to BMI. Conclusions: Overall obesity and abdominal obesity are substantially prevalent in the studied adolescent girls. Further studies are needed to identify risk factors for the obesity in our studied population. Findings emphasize the importance of establishing community-based interventions in order to prevent the development of adolescent obesity and its complications in adulthood.
\end{abstract}

KEYWORDS: Adolescent, body mass index, abdominal obesity, girls

\section{INTRODUCTION}

Chronic non-communicable diseases such as cardiovascular diseases and diabetes are the leading cause of death worldwide. ${ }^{1}$ According to World Health Organization estimates, by the year 2020, noncommunicable diseases will account for approximately three-quarters of all deaths in the developing world. ${ }^{2}$ Obesity is a major risk factor for chronic diseases and plays a central role in the "insulin resistance" or "metabolic syndrome", which includes hyperinsulinemia, hypertension, hyperlipidemia, type 2 diabetes mellitus, and an increased risk of atherosclerotic cardiovascular disease. ${ }^{3}$

Corresponding author:

Maryam Rafraf

Associate professor,

Nutritional Research Center,

Department of Community Nutrition,

Faculty of Health \& Nutrition,

Tabriz University of Medical Sciences,

Tabriz, Iran.

Phone: 00984113357581-4

Fax: 00984113340634

E-mail: rafrafm@tbzmed.ac.ir
Overweight and obesity are among important medical problem between adults, children and adolescents in developed and developing countries. ${ }^{4-6}$ The prevalence of childhood obesity has increased over the last two decades. ${ }^{7}$ Urbanization, unhealthy diets, and increasingly sedentary lifestyles have contributed to increase the prevalence of childhood obesity, particularly in developing countries. ${ }^{8}$ Previous studies indicated that the process of atherosclerosis starts at an early age and is already linked to obesity and other components of the metabolic syndrome in childhood. ${ }^{9}$ Increasing obesity prevalence among children and adolescents is one of the most important public health problems. ${ }^{4}$

According to the first results of the Iranian national study conducted by Motlagh et al., $2.6 \%$ and $11.1 \%$ of high-school students (14-18 years) were overweight and at risk for overweight based on the body mass index (BMI) status, respectively.10 The prevalence of weight disorders was significantly different in different districts. ${ }^{10}$ In another study by Mirhosseini et al. in Mashad, Iran, $14.6 \%$ and $3.4 \%$ of adolescent girls were classified as overweight and obese, respectively. Enlarged waist circumference (WC) greater than 80 $\mathrm{cm}$, was seen in $9.5 \%$ of subjects. ${ }^{11}$ 
WC has been shown to correlate well with intraabdominal fat mass, ${ }^{12}$ and abdominal obesity is considered as an independent predictor of several risk factors and morbidity. ${ }^{13-15}$ Therefore, early identification and treatment of children or adolescents with central adiposity is very important due to metabolic complications. ${ }^{4,14}$

Considering that adiposity generally tracks from childhood into adulthood, and is associated with adult health outcomes, and with attention to the scarcity of studies of the subject in our country, the present study has been carried out in order to estimate the prevalence of overall obesity and abdominal obesity by commonest anthropometric measurements such as: BMI, waist-to-hip ratio (WHR),WC and waist-to-height ratio (WHtR) in Iranian adolescent girls attending high school in Tabriz, the capital of east Azerbaijan, in northwest Iran.

\section{METHODS}

The ethical committee of Tabriz University of Medical sciences approved the study protocol. In this cross-sectional study, a sample of 985 girls (14 to 17 years old) was selected from among high-school students in Tabriz, Iran by a two-stage stratified random-sampling technique. Written consent was obtained from the students and their parents. Information on general characteristics was obtained by interview. Subjects with a history of disease, including diabetes, heart disease, kidney disease, gastrointestinal disease, or use of any medication, were excluded from the study. Data were gathered by two trained nutritionists. One of the nutritionists interviewed the subjects and measured their weight and height, and the other performed waist and hip measurements.

Body weight was measured using calibrated beam scales (Seca, Germany) and was recorded to the nearest $0.5 \mathrm{~kg}$. The subjects were measured barefoot wearing light clothing. Height was measured using a mounted tape (nonelastic) with the subject's arms hanging freely at her sides and was recorded to the nearest $0.5 \mathrm{~cm}$. BMI was calculated as the weight in kilograms divided by the height in meters squared. Underweight, healthy weight, overweight, and obesity were defined according to the International BMI cut-off points for adolescents. Overweight for girls aged 14 to $14.9,15$ to $15.9,16$ to 16.9 , and 17 to 17.9 years is defined as $\mathrm{BMI} \geq 23.66$ to $<28.87$, $\geq$
24.17 to $<29.29$, $\geq 24.54$ to $<29.56$, and $\geq 24.85$ to $<$ 29.84 , respectively. Obesity is defined as BMI $\geq 28.87$, $\geq 29.29$, $\geq 29.56$, and $\geq 29.84$, respectively. These cutoffs are based on BMI percentiles but use data pooled from six large, nationally representative surveys. ${ }^{16,17}$

WC was measured at the minimum circumference between the iliac crest and the rib cage over light clothing, and hip circumference $(\mathrm{HiC})$ was recorded at the maximum circumference over the buttocks, using a nonelastic flexible tape measure, without any pressure to the body surface in a standing position. Measurements were recorded to the nearest $0.1 \mathrm{~cm}$. Abdominal obesity was diagnosed when the $W C \geq$ the age -and sex -specific 90th percentile for this population, ${ }^{18,19}$ or $\mathrm{WHR}>0.80$ or $\mathrm{WHtR} \geq 0.50 .{ }^{20}$

Statistical analysis was performed with SPSS for Windows, version 11.5. The results were considered statistically significant if $p<0.05$. The chi-square test and Pearson's coefficient analysis were used to determine the relationship between WC, WHR, and WHtR and BMI status. Correlation between anthropometric measurements and age were also evaluated by Pearson's coefficient test.

\section{RESULTS}

Table 1 shows the mean of weight, height, BMI, WC, $\mathrm{HiC}, \mathrm{WHR}$, and WHtR of the study population according to age groups. Mean age of subjects was $15.67 \pm$ 1.01 years. Pearson's correlation coefficient analysis showed no significant correlation between any anthropometric measures and age.

Table 2 shows the distribution of subjects based on BMI, WC, WHR, and WHtR status. The prevalence rates of obesity and overweight were $2.8 \%$ and $16.4 \%$, respectively. According to WC, WHR, and WHtR classifications, $13.2 \%, 14 \%$, and $18.2 \%$ of subjects had abdominal obesity, respectively.

There were significant relationship between WC, WHR, and WHtR and BMI status of subjects by the chi-square test, as WC, WHR, and WHtR increased with increasing $\mathrm{BMI}\left(\mathrm{X}^{2}=436.67, \mathrm{P}=.001 ; \mathrm{X}^{2}=122.19\right.$, $\mathrm{P}=.001$ and $\mathrm{X}^{2}=446.23, \mathrm{P}=.001$, respectively). In Pearson's correlation coefficient analysis, there were also significant positive correlations between WC, WHR, and WHtR and BMI $(r=.86, p=.001 ; r=.50, p=.001$ and $r=.87, p=.001$, respectively; data not shown). 
Table 1. Descriptive characteristics of subjects

\begin{tabular}{llllllll}
\hline Age $(\mathrm{y})$ & Weight (kg) & Height (cm) & BMI (kg/m2) & WC (cm) & HiC (cm) & WHR & WHtR \\
& Mean (s.d) & Mean (s.d.) & Mean (s.d.) & Mean(s.d.) & Mean (s.d.) & Mean (s.d.) & Mean (s.d.) \\
\hline $14-14.9(\mathrm{n}=143)$ & $53.29(9.20)$ & $158.89(5.83)$ & $21.11(3.52)$ & $69.96(8.70)$ & $91.61(7.48)$ & $0.76(0.07)$ & $0.44(0.06)$ \\
$15-15.9(\mathrm{n}=283)$ & $53.78(10.2)$ & $159.66(5.71)$ & $21.07(3.7)$ & $70.25(8.70)$ & $92.39(7.48)$ & $0.76(0.05)$ & $0.44(0.05)$ \\
$16-16.9(\mathrm{n}=311)$ & $54.70(10.12)$ & $159.22(5.81)$ & $21.56(3.67)$ & $71.01(8.20)$ & $93.53(7.20)$ & $0.76(0.5)$ & $0.45(0.05)$ \\
$17-17.9(\mathrm{n}=248)$ & $54.69(9.32)$ & $159.47(5.2)$ & $21.35(3.67)$ & $70.75(8.43)$ & $94.09(6.98)$ & $0.76(0.05)$ & $0.44(0.05)$ \\
Total $(\mathrm{n}=985)$ & $54.23(9.82)$ & $159.36(5.64)$ & $21.35(3.67)$ & $70.75(8.43)$ & $93.06(7.2)$ & $0.76(0.05)$ & $0.44(0.05)$
\end{tabular}

Table 2. Distribution of subjects based on overall obesity and abdominal obesity by BMI category

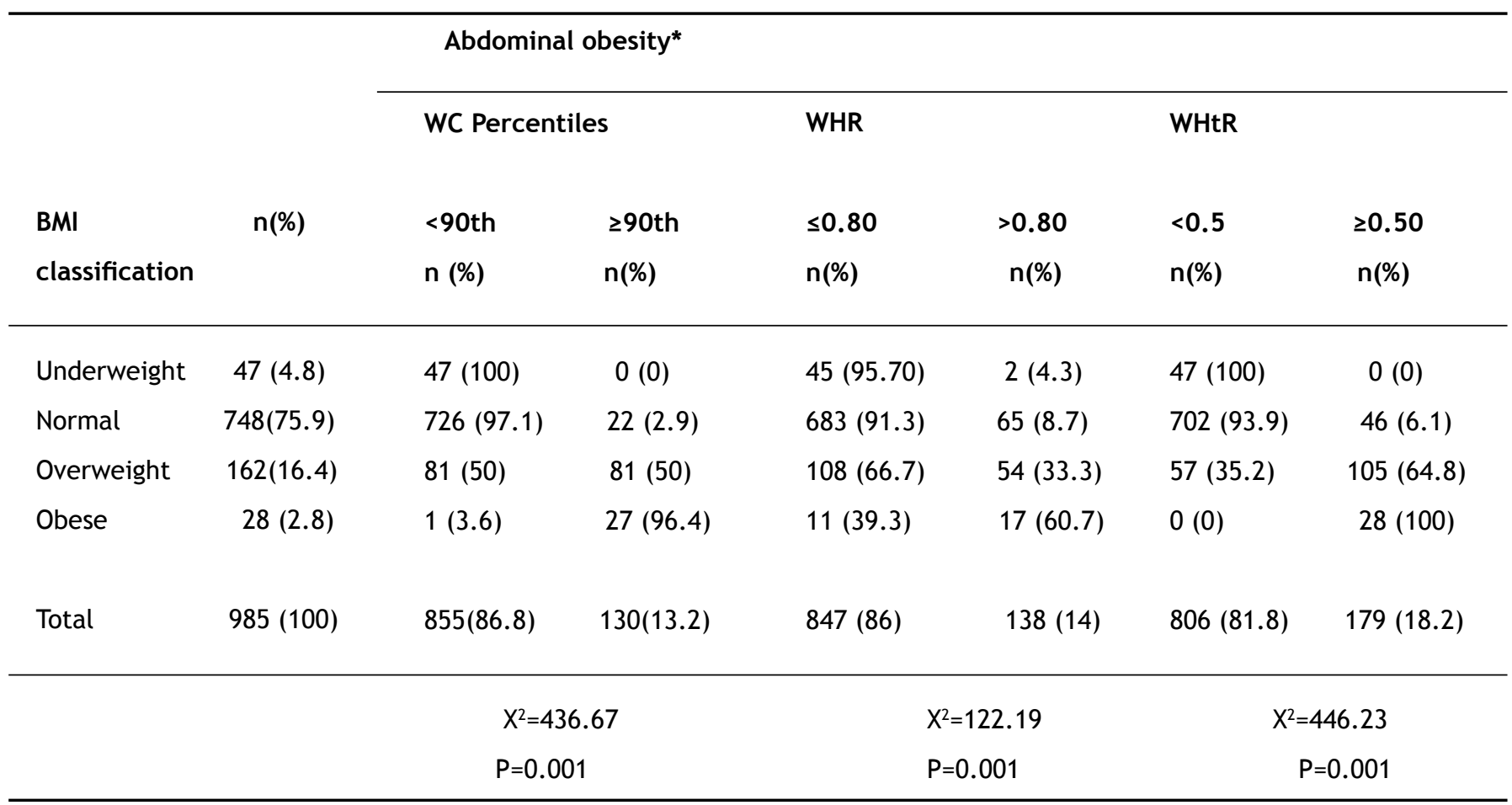

*Abdominal obesity: waist circunmference $\geq 90$ th for age and sex; WHR $>0.80$; WHtR $\geq 0.50$, WC:

Waist circumference, WHR: waist to hip ratio, WHtR: weight to height ratio 


\section{DISCUSSION}

The prevalence rates of obesity and overweight were $2.8 \%$ and $16.4 \%$, respectively (Table 2 ). These results were similar to those of our previous study on high school girls in Tabriz, in 2001.21 The national survey conducted in Iran, among 21,111 school students aged 6-18 years in 2003-2004, showed that according to US Centers for Disease Control and Prevention (CDC) and International Obesity Task Force (IOTF) cut offs, the prevalence of obesity was $4.5 \%$ and $2.9 \%$ and the rates for overweight were $8.82 \%$ and $11.3 \%$, respectively..$^{10}$ The prevalence of obesity in our studied subjects was similar to the study mentioned above; however, the prevalence of overweight was higher than that of study.

In a study among Mexican adolescent girls, based on CDC cutoffs, the prevalence of overweight and obesity were $21 \%$ and $9 \%$, respectively. ${ }^{22}$ Among adolescent (male \& female) aged 11-17 years in Greece, the prevalence of overweight and obesity were $19.1 \%$ and $3.2 \%$, respectively. ${ }^{23}$ Among girls aged $13-18$ years in India, the age-adjusted prevalence of overweight was $15.8 \%,{ }^{24}$ and within Tunisian adolescent girls was found to be $16 \%{ }^{25}$ In studies among Egyptian adolescent girls, ${ }^{22}$ Saudi Arabian youths over 12 years of age ${ }^{26}$ and adolescent girls in the United Arab Emirates, ${ }^{27}$ using the CDC cutoffs for BMI, $18 \%$ \& $8 \%, 13.8 \%$ \& $20.5 \%$, and $14 \%$ \& $9 \%$ of subjects were overweight and obese, respectively. Based on IOTF cutoffs, the overall obesity in Bahrain girls aged $12-17$ years were $18 \%,{ }^{28}$ in Qatar, $18.9 \%$ \& $4.7 \% 29$ and in Turkey $10.6 \%$ \& $2.1 \%$ of adolescent girls were overweight and obese, respectively. ${ }^{30}$ In Dalian, China, $10.4 \%$ of girls aged 9-16 were overweight based on CDC cutoffs for BMI. ${ }^{31}$

Comparison of our results with studies in other countries shows that the prevalence of obesity and overweight in Tabriz high school girls is lower than some other countries such as Mexico and Greece, ${ }^{22,23}$ and that of some developing countries, including Bahrain and Qatar. ${ }^{28,29}$ However, the prevalence of overweight in our studied subjects was higher from countries such as China and Turkey, ${ }^{30,31}$ and was somewhat similar to rates in India and Tunisia. ${ }^{24,25}$ In addition to the overall obesity, WC is a useful measure of the abdominal obesity that is more closely related to cardiovascular risk and metabolic syndrome than overall obesity. ${ }^{32}$ BMI fails to distinguish between muscle and fat, and seems therefore, a poor proxy for central fatness. ${ }^{14}$

According to the results (table 2), 13.2\%, 14.0\%, and $18.2 .0 \%$ of subjects had abdominal obesity, based on WC, WHR and WHtR classifications, respectively. In a study by Esmaillzadeh et al. on 1623 girls aged 10-19 years in Tehran (capital city of Iran), the prevalence of abdominal obesity was $10.1 \%$ based on WC categorized by age and sex specific $90^{\text {th }}$ percentile for their population. ${ }^{9}$ In another study performed in Mashhad, Iran, $9.5 \%$ of adolescent girls aged $15-17$ years had $W C>80 \mathrm{~cm}$. 11The rate of abdominal obesity in our studied population is higher than above studies. No other published data are available about abdominal obesity in Iranian adolescent girls.

Abolfotouh et al. showed prevalence of abdominal obesity in \% $16.1 \%, 4.5 \%$ and $16.7 \%$ in 1500 Egyptian males and females aged $11-19$ years according to WHR, WC and WHtR, respectively. ${ }^{7}$ McCarfhy HD and Ashwell $M$ reported WHtR exceeded the 0.50 in $11.7 \%$ of adolescent girls aged 11-16 years in UK children in 1997. ${ }^{20}$ In 279 Swedish girls aged $15.6 \pm 0.4$ years, high-risk waist circumference was detected in $30.1 \%$ of subjects. ${ }^{33}$

Children from different populations vary in their rate of proportional growth and in fat patterning. ${ }^{12,34}$ Ethnicity is one of the important factors influencing body-fat distribution via heterogeneity in total mass and composition of skeletal muscles, subcutaneous and intra-abdominal adipose tissue, and bone. Less skeletal muscle mass and pelvic skeleton dimensions are seen in Asian, particularly those who suffered childhood malnutrition, and may affect waist and hip circumferences. ${ }^{35}$ Several epidemiologic studies in Asian population have shown that Asian, including Chinese, Taiwanese, Indians and Koreans have higher amounts of body fat at lower BMls and waist circumferences than do Western populations such as US whites and blacks, perhaps leading to the greater prevalence of cardiovascular disease risk factors at lower BMIs in Asian populations than in Western populations. ${ }^{36}$

In our sample (table 2), the majority of obese and overweight subjects had an abdominal obesity. WC, WHR, and WHtR were related strongly to BMI. WC and WHtR had the highest relationship with BMI. The percent of abdominal obesity based on WHtR in our study, also showed the highest prevalence comparing to WC or WHR (table 2). WHtR has been proposed as an alternative, conveniently age-independent measure of cardiovascular risk. It might be a better predictor of risk for cardiovascular disease than BMI or WC for the following reasons (1) WHtR is more highly correlated with visceral fat mass- and clustering of cardiovascular risk factors in children- and adults (2) WHtR may be a more accurate tracking indicator of fat distribution and accumulation by age, because it accounts for the growth in both WC and height over age, particularly in children and adolescent; and (3) the value of WHtR is free of measurement units and is in a close agreement between males and females at each age group. Thus, WHtR may be a potentially useful surrogate measure for abdominal obesity across different age, gender, or racial/ethnic subpopulations. ${ }^{37,38}$

Our results are of particular concern, because visceral adiposity, measured by WC and WHtR, increases the risk for obesity- associated morbidity and mortality in children and adults. ${ }^{38}$ In a national study by Motlagh and et al. an increased trend in the prevalence of Cardiovascular disease risk factors, especially dyslipidemia and overweight among Iranian children and adolescents was reported. ${ }^{10}$ 
Over the past decades, there has been increasing evidence that the structure of dietary intakes and the prevalence of obesity around the developing world have been changing at an increasing rapid pace. ${ }^{6}$ Countries of the Middle East are believed to have the highest dietary energy surplus among developing countries. Iran, among other countries of the Eastern Mediterranean Region, has undergone a rapid transition in patterns of nutrition. This follows the rapid change in fertility and mortality patterns and increasing urbanization, which has led to considerable imbalance in food composition favoring low-nutrient diets and over-consumption among more than one-third of households. ${ }^{10}$ Increases in energy intake, portion sizes, sweetened beverage intake, and declines in sufficient vigorous physical activity, despite declines in sedentary behaviors (e.g. hours of television viewing) likely contributed to obesity in children and adolescents in different populations. ${ }^{38}$ We did not evaluate such lifestyle behaviors, so they are considered as limitations of our study. Other studies are needed to demonstrate causality of risk factors on obesity in our community.

As a conclusion, our study provides evidence showing a substantial prevalence of overall and abdominal obesity in adolescent girls of our community. Measurement of BMI, WC and especially WHtR may help to identify adolescent girls with risk factors for chronic disease in adulthood. It is suggested to establish surveillance programs and preventive strategies in studied population to reduce the likely medical and psychological costs of the increase in obesity in young people. Findings emphasize the need to encourage healthy lifestyles, particularly healthy nutrition and increasing physical activity for adolescent girls.

\section{ACKNOWLEDGMENT}

We thank the Office of Nutritional Research Center of Tabriz University of Medical Sciences, Tabriz, Islamic Republic of Iran for the financial support; the school headmistresses and students who participated in this study.

\section{REFERENCES}

1. Yach D, Hawkes C, Gould CL, Hofman KJ. The global burden of chronic diseases: overcoming impediments to prevention and control. JAMA 2004; 291:2616-22.

2. Global strategy for non-communicable disease prevention and control (draft). Geneva, World Health Organization 1997 (WHO/NCD/GS/97.1).

3. Sinaiko AR, Jacobs DR, Steinberger J, et al. Insulin resistance syndrome in childhood: associations of the euglycemic insulin clamp and fasting insulin with fatness and other risk factors. J Pediatr 2001; 139:700-7.

4. Hatipoglu N, Ozturk A, Mazicioglu MM, et al. Waist circumference percentiles for 7 - to 17-year-old Turkish children and
5. Flodmark CE, Lissau I, Moreno LA, Pietrobelli A, Widhalm K. New insights into the field of children and adolescents' obesity: the European perspective. Int J Obes 2004; 28:1189-96.

6. Popkin BM, Gordon-Larsen P. The nutrition transition: worldwide obesity dynamics and their determinants. Int J Obes 2004; 28:2-9.

7. Abolfotouh MA, Sallam SA, Mohammed MS, Loutfy AA, Hasab A. Prevalence of elevated blood pressure and association with obesity in Egyptian school adolescents. Int J Hypertens 2011; 8:952537.

8. Zimmet P, Alberti K George MM, et al. The metabolic syndrome in children and adolescents - an IDF consensus report. Pediatr Diabetes 2007; 8:299-306.

9. Esmaillzadeh A, Mirmiran P, Azadbakht L, Etemadi A, Azizi F. High prevalence of the metabolic syndrome in Iranian adolescents. Obesity 2006; 14:377-82.

10. Motlagh ME, Kelishadi R, Ardalan G, et al. Rationale, methods and first results of the Iranian national programme for prevention of chronic diseases from childhood: CASPIAN Study. East Mediterr Health J 2009; 15:302-14.

11. Mirhosseini NZ, Yusoff NA, Shahar S, et al. Prevalence of the metabolic syndrome and its influencing factors among adolescent girls in Mashhad, Iran. Asia Pac J Clin Nutr 2009; 18:131-6.

12. MCCarthy HD, Jarrett KV, Crawley HF. The development of waist circumference percentiles in British children aged $5.0 \pm$ 16.9 y. Eur J Clin Nutr 2001; 55:902-7. 13. Bahrami H, Sadatsafavi M, Pourshams A, et al. Obesity and hypertension in an Iranian cohort study; Iranian women experience higher rates of obesity and hypertension than American women. BMC Public Health 2006; 6:158.

14. Fredriks AM, Buuren SV, Fekkes $M$, Verloove-Vanhorick SP, Wit JM. Age and references for waist circumference, hip circumference and waist-hip ratio in Dutch children useful in clinical practice? Eur J Pediatr 2005; 164:216-22.

15. Caprio S, Hyman LD, McCarthy S, et al. Fat distribution and cardiovascular risk factors in obese adolescent girls: importance of the intraabdominal fat depot. Am J Clin Nutr 1996; 64: 12-17.

16. Cole TJ, Flegal KM, Nicholls D, Jackson AA. Body mass index cut offs to define thinness in children and adolescents: Inter national survey. BMJ 2007; 335:194.

17. Cole TJ, Bellizzi MC, Flegal KM, Dietz WH. Establishing a standard definition for child overweight and obesity worldwide: International survey. BMJ 2000; 320:1240-3. 18. Kelishadi R. Prevention and control of 
childhood Obesity: today better than tomor row. 1st Ed., Isfahan University of Medical Sciences, 2010, p: 38.

19. Kelishadi R, Gouya MM, Ardalan G, et al. First reference curves of waist and hip circumferences in an Asian population of youths: CASPIAN Study. J Trop Pediatr 2007; 53:158-64.

20. McCarthy HD, Ashwell M. A study of central fatness using waist-to height ratios in UK children and adolescents over two decades supports the simple message - 'keep your waist circumference to less than half your height'. Int J Obes 2006; 30:988-92.

21. Gargari BP, Behzad MH, Ghassabpour S, Ayat A. Prevalence of overweight and obesity among high-school girls in Tabriz, Iran, in 2001. Food Nutr Bull 2004; 25:288-91.

22. Salazar-Martinez E, Allen B, Fernandez-Ortega $C$, et al. Overweight and obesity status among adolescents from Mexico and Egypt. Arch Med Res 2006; 37:535-42.

23. Krassas GE, Tzotzas T, Tsametis C, Konstantinidis T. Prevalence and trends in overweight and obesity among children and adolescents in Thessaloniki, Greece. J Pediatr Endocrinol Metab 2001; 14:1319-26.

24. Ramachandran A, Snehalatha C, Vinitha R, et al. Prevalence of overweight in urban Indian adolescent school children. Diabetes Res Clin Pract 2002; 57:185-90.

25. Ghannem H, Darioli R, Harrabi I, et al. Epidemiology of cardiovascular risk factors among schoolchildren in Sousse, Tunisia. J Cardiovasc Risk 2001; 8:87-91.

26. Al-Rukban MO. Obesity among Saudi male adolescents in Riyadh, Saudi Arabia. Saudi Med J 2003; 24:27-33.

27. Al-Hourani HM, Henry CJ, Lightowler HJ. Prevalence of overweight among adolescent females in the United Arab Emirates. Am J Hum Biol 2003; 15:758-64.

28. Al-Sendi AM, Shetty P, Musaiger AO. Prevalence of overweight and obesity among Bahraini adolescents: a comparison between three different sets of criteria. Eur $\mathrm{J}$ Clin Nutr 2003; 57:471-4.

29. Bener A. Prevalence of obesity, overweight, and underweight in Qatari adolescents. Food Nutr Bull 2006; 27:39-45.

30. Oner N, Vatansever U, Sari A, et al. Prevalence of underweight, overweight and obesity in Turkish adolescents. Swiss Med Wkly 2004; 134:529-33.

31. Zhou H, Yamauchi T, Natsuhara K, et al. Overweight in urban schoolchildren assessed by body mass index and body fat mass in Dalian, China. J Physiol Anthropol 2006; 25:41-8.
32. Pedrosa C, Correia F, Seabra D, et al. Prevalence of overweight and obesity among 7-9-year-old children in Aveiro, portugal: comparison between IOTF and CDC references. Public Health Nutr 2009; 14:14-9.

33. Ortega FB, Ruiz JR, Sjostrom M. Physical activity, overweight and central adiposity in Swedish children and adolescents: the European Youth Heart Study. Int J Behav Nutr Phys Act 2007; 4:61.

34. Tybor DJ, Lichtenstein AH, Dallal GE, Daniels $\mathrm{SR}$, Must A. Racial differences in central adiposity in a longitudinal cohort of black and white adolescent females. BMC Pediatr 2010; $10: 2$.

35. Misra A, Vikram NK, Gupta R, et al. Waist circumference cutoff points and action levels for Asian Indians for identification of abdominal obesity. Int J Obes 2006; 30: 106-11.

36. Wildman RP, Gu D, Reynolds K, Duan X, He J. Appropriate body mass index and waist circumference cutoffs for categorization of overweight and central adiposity among Chinese adults. Am J Clin Nutr 2004; 80: 1129-36.

37. Sung RT, So HK, Choi KC, et al. Waist circumference and waist-to-height ratio of Hong Kong Chinese children. BMC Public Health 2008; 8:324-33.

38. Li C, Ford ES, Mokdad AH, Cook S. Recent trends in waist circumference and waistheight ratio among US children and adolescents. Pediatrics 2006; 118:e1390-8. 